\title{
Achieving Symmetric Pareto Nash Equilibria using Biased Replicator Dynamics
}

\author{
Kiran K. Somasundaram and John S. Baras
}

\begin{abstract}
Achieving the Nash equilibria for single objective games is known to be a computationally difficult problem. However there is a special class of equilibria called evolutionary robust equilibria which can be obtained through a special type of evolutionary dynamics called the replicator dynamics. This dynamics has special properties over the simplex, which has been studied in optimization theory to solve several combinatorial problems. In this work, we consider the essentially hard combinatorial optimization problem of computing the equilibria in games with multiple objectives. We extend the notion of replicator dynamics to handle such games. We establish proofs of dynamic stability of this modified replicator dynamics and present their relation to the Pareto Nash equilibria in multiobjective games.
\end{abstract}

\section{INTRODUCTION}

In conventional game theoretic problems, it is usually assumed that the decision makers usually make their decisions based on a scalar payoff. But in many practical problems in economics and engineering, decision makers usually deal with multiple objectives or payoffs. In these problems, one needs to consider a vector payoff function. The notion of vector payoffs was originally introduced by Blackwell [2] and later by Contini [3]. A more rigorous model for zerosum games and games against nature was studied by Zeleny [12]. In [12], Zeleny solves the multiple objective zero-sum game using multi-parametric criteria linear programming. In the preceding work by Contini, a non-zero-sum version of the Multi-Objective Game (MOG) was introduced. A general version concerning the n-person MOG in the noncooperative setting was introduced in [4]. A further extension to cooperative games and hybrid games was introduced by Zhao in [9]. Then notions of Pareto Nash strategies were reborn in the works by Zelikovsky [5] where the authors provide algorithms to obtain the Pareto Nash Equilibria.

However, we observe that most of these algorithms are only existential in nature and do not offer any constructive means to achieve the equilibria. As far as the complexity of the non-zero sum games is concerned, the vector payoff games can be shown to be as hard as their single objective counterparts. In this work, we develop a continuous time dynamics to achieve a special set of equilibria called the evolutionary equilibria. This special type of equilibria was introduced in evolutionary biology by Maynard Smith [16], within the context of single objective payoffs. However in many practical scenarios, single objective games become handicapped in capturing the tradeoff relations between the

Institute for Systems Research and Department of Electrical and Computer Engineering, University of Maryland, College Park, MD 20742 kirans@umd.edu, baraseisr.umd.edu various payoffs that players perceive in an evolutionary sense. To the best of our knowledge, there has been no previous work in this area for vector payoffs. We feel that the theory of MOG has significant potential to model several practical scenarios especially in the context of networked controls and communications. For examples we refer the reader to [1].

This paper is organized as follows. In section II, we introduce the notion of equilibria in a MOG. We also provide a novel proof to establish existence of such an equilibrium. In section III, we shift focus to two player MOGs, which would be the topic of interest for the rest of the paper. In this section, we show the equivalence of the player's best strategy against opponents to a parametric multi-objective linear program. In section IV, we show that this relation is useful in constructing the tradeoff weights among the various payoffs. Finally, in section V, we present the continuous time dynamics, called the biased replicator dynamics, to achieve the evolutionary stable set of strategies.

\section{PARETO NASH EQUILIBRIUM}

As in single objective games, the notion of equilibrium can be defined in terms of unfruitful deviation from the equilibrium strategies. In the MOG setting, this can be interpreted as the following:

"Deviations from the equilibrium strategies do not offer any gains to any of the payoff functions for any of the players."

We introduce the notion of orders in $\mathbb{R}^{l}$ in Table I to compare vector payoffs. These orders are commonly used in the multi-criteria optimization literature.

\begin{tabular}{|l|l|l|}
\hline Notation & Definition & Name \\
\hline$x \geq y$ & $x_{i} \geq y_{i} \quad i=1,2, . ., l$ & Weak component-wise order \\
$x>y$ & $x_{i} \geq y_{i} \quad i=1,2, . ., l$ and $x \neq y$ & Component-wise order \\
$x \gg y$ & $x_{i}>y_{i} \quad i=1,2, . ., l$ & Strict component-wise order \\
\hline \multicolumn{3}{c}{ TABLE I } \\
\hline \multicolumn{3}{c}{ TABLE OF ORDERS IN $\mathbb{R}^{l}$}
\end{tabular}

To rigorously define the Pareto Nash Equilibria, we need to introduce certain notation and terminology that is prevalent in the game theoretic literature.

\section{A. MATHEMATICAL NOTATION}

We follow the notations used in the book by Weibull [10]. We consider finite games in normal form. Let $I=$ $\{1,2, \cdots, n\}$ be the set of players, where $n \geq 2$. For each player $i \in I$, let $S_{i}$ be her finite set of pure strategies. Let the pure strategies set of player $i \in I$ be $S_{i}=\left\{e_{i}^{1}, e_{i}^{2}, \cdots, e_{i}^{m_{i}}\right\}$, where $m_{i} \geq 2$. The vector $s$ of pure strategies, $s=$ 
$\left[s_{1}, s_{2}, \cdots, s_{n}\right]$, where $s_{i}$ is the pure strategy of any player $i$, is called the pure strategy profile. The pure strategies profiles live in the Cartesian product space $S=\times_{i} S_{i}$.

For any strategy profile $s \in S$ and player $i \in I$, let $\underline{\pi}_{i}(s) \in$ $\mathbb{R}^{l_{i}}$ be the $l_{i}$ dimensional vector payoff function for player $i$ when all the players play a strategy profile $s$. The combined payoff function $\underline{\pi}: S \rightarrow \times_{i} \mathbb{R}^{l_{i}}$ is the collection $\underline{\pi}(s)=$ $\left(\underline{\pi}_{1}(s), \underline{\pi}_{2}(s), \cdots, \underline{\pi}_{n}(s)\right)$.

A mixed strategy for player $i$ is a probability distribution over her set $S_{i}$ of pure strategies. Let $x_{i}$ denote the mixed strategy for player $i$. Then $x_{i}$ lives in the $\left(m_{i}-1\right)$ dimensional unit simplex $\Delta_{i}$, where

$$
\Delta_{i}=\left\{x_{i} \in R_{+}^{m_{i}} \quad: \quad \sum_{h=1}^{m_{i}} x_{i h}=1\right\}
$$

These are probability distributions over the pure strategies, which are represented by the vertices of $\Delta_{i}, e_{i}^{1}=$ $[1,0, \cdots, 0], e_{i}^{2}=[0,1, \cdots, 0], \cdots, e_{i}^{m_{i}}=[0,0, \cdots, 1]$. Thus, the mixed strategies can be alternatively represented as $x_{i}=\sum_{h=1}^{m_{i}} x_{i h} e_{i}^{h}$. A mixed strategy profile is a vector $x=\left[x_{1}, x_{2}, \cdots, x_{n}\right]$ that lives in $\Theta=\times_{i} \Delta_{i}$. Let $C\left(x_{i}\right)$ be the support of the vector $x_{i}$; i.e $C_{i}=\left\{\begin{array}{l|l}h & \mid x_{i h}>0\end{array}\right\}$.

The mixed strategies payoff functions are given by $\underline{u}_{i}(x)=\sum_{s \in S} x(s) \underline{\pi}_{i}(s)$, where $x(s)=\prod_{i} x_{i s_{i}}$. If player $j$ 's strategy in the strategy profile $x$ is replaced by another strategy $y_{j}$ we denote the new profile by $\left(y_{j}, x_{-j}\right)$. Here $x_{-j}$ represents the strategy profile of all players other than player $j$. Then the payoff function can be expressed as

$$
\underline{u}_{i}(x)=\sum_{k=1}^{m_{j}} \underline{u}_{i}\left(e_{j}^{k}, x_{-j}\right) x_{j k}
$$

It should be noted that $\underline{u}_{i} \in \mathbb{R}^{l_{i}}$. i.e., the vector payoff function is expressed as $\underline{u}_{i}(x)=\left[u_{i}^{1}(x) u_{i}^{2}(x) \cdots u_{i}^{l_{i}}(x)\right]$. Each component function $u_{i}^{k}, \quad k \in\left\{1,2, \cdots, l_{i}\right\}$ is a multilinear mapping that is linear in each vector component $x_{j} \in \Delta_{j}$. The collection of payoff vectors for all $i \in I$ is called the combined mixed strategy payoff function, and is denoted by $\underline{u}(x)=\left[\underline{u}_{1}(x), \underline{u}_{2}(x), \cdots, \underline{u}_{n}(x)\right]$.

\section{B. PARETO REPLIES AND PARETO DOMINATING REPLIES}

We next introduce our Pareto reply and Pareto dominating reply that are analogous to Nash's best reply and better response reply of a single objective game.

Definition The Pareto Reply of player $i \in I$ for the strategy profile $x_{-i}$ of the rest of the players is defined as that strategy $x_{i} \in \Delta_{i}$ such that the strategy profile $\left(x_{i}, x_{-i}\right)$ is Pareto optimal with respect to the vector payoff function $\underline{u}_{i}\left(., x_{-i}\right)$.

The Pareto reply correspondence

$\beta_{i}^{P}: \times_{k \neq i} \Delta_{k} \rightarrow 2^{\Delta_{i}}$ is given by

$$
\begin{array}{r}
\beta_{i}^{P}\left(x_{-i}\right)=\left\{x_{i} \in \Delta_{i} \quad \mid \quad \nexists z \in \Delta_{i}\right. \\
\text { such that } \underline{u}_{i}\left(z, x_{-i}\right)>\underline{u}_{i}\left(x_{i}, x_{-i}\right\}
\end{array}
$$

where $>$ is the component-wise order defined in $\mathrm{Ta}$ ble I. The combined Pareto reply correspondence of the strategy profile $x$ is then given by $\beta^{P}(x)=$ $\left(\beta_{1}^{P}\left(x_{-1}\right), \beta_{2}^{P}\left(x_{-2}\right), \cdots, \beta_{n}^{P}\left(x_{-n}\right)\right)$.

Definition A strategy profile $x^{*}=\left[x_{1}^{*}, x_{2}^{*}, \cdots, x_{n}^{*}\right]$ is called a Pareto Nash equilibrium for the MOG if $x^{*} \in \beta^{P}\left(x^{*}\right)$. The set of all Pareto Nash equilibria is denoted by $\Theta^{P N E}$.

In other words, a Pareto equilibrium is a fixed point of the Pareto reply correspondence. The Kakutani fixed point theorem cannot be invoked to prove the existence of such an equilibrium here because the Pareto replies do not necessarily form a convex set. A detailed reference to these fixed point problems is available in [6]. In the current game theoretic literature, there are several complicated proofs for existence of equilibria in various topological spaces. We give an alternative proof for the existence of the Pareto Nash equilibria that is solely motivated from Nash's original work and Pareto optimality.

Nash, in his seminal work on non-cooperative games [7], [8], introduced the notion of better responses, which has motivated the study of several similar dynamics in the literature [10]. However, it should be mentioned that Nash used the better response maps to prove the existence of a non-cooperative equilibrium for single objective games. In the same spirit, we define the Pareto dominating reply. For an arbitarily small $\epsilon>o$, we define

$$
\begin{aligned}
c_{i}^{h}(x)=\quad & \min \left(\left(u_{i}^{1}\left(e_{i}^{h}, x_{-i}\right)-u_{i}^{1}(x)+\epsilon\right)^{+},\right. \\
& \left(u_{i}^{2}\left(e_{i}^{h}, x_{-i}\right)-u_{i}^{2}(x)+\epsilon\right)^{+}, \cdots, \\
& \left.\left(u_{i}^{l_{i}}\left(e_{i}^{h}, x_{-i}\right)-u_{i}^{l_{i}}(x)+\epsilon\right)^{+}\right) \\
& \max \left(\left(u_{i}^{1}\left(e_{i}^{h}, x_{-i}\right)-u_{i}^{1}(x)\right)^{+},\right. \\
& \left(u_{i}^{2}\left(e_{i}^{h}, x_{-i}\right)-u_{i}^{2}(x)\right)^{+}, \cdots, \\
& \left.\left(u_{i}^{l_{i}}\left(e_{i}^{h}, x_{-i}\right)-u_{i}^{l_{i}}(x)\right)^{+}\right)
\end{aligned}
$$

The Pareto dominating reply map $D^{P}: \Theta \rightarrow \Theta$ is defined by

$$
x_{i h}^{\prime}=\frac{x_{i h}+c_{i}^{h}(x)}{1+\sum_{k \in S_{i}} c_{i}^{k}(x)} \quad \forall h \in S_{i} \quad \forall i \in I
$$

Lemma 2.1: The map $D^{P}: \Theta \rightarrow \Theta$ has a fixed point $x^{f}$.

Proof: The map $D^{P}$ is continuous, and the set $\Theta$ is a compact convex set. Then by Brouwer's fixed point theorem([6]), there exists a fixed point $x^{f}$ for $D^{P}$ in $\Theta$.

Lemma 2.2: For any strategy profile $x, \forall i \in I$, and $\forall p \in\left\{1,2, \cdots, l_{i}\right\}, \exists h(p) \in C\left(x_{i}\right)$ such that $u_{i}^{p}\left(e_{i}^{h(p)}, x_{-i}\right) \leq u_{i}^{p}(x)$.

Proof: Suppose there exists no $h \in C\left(x_{i}\right)$ such that $u_{i}^{p}\left(e_{i}^{h}, x_{-i}\right) \leq u_{i}^{p}(x)$. Then for all $h \in C\left(x_{i}\right)$ we have

$$
\begin{aligned}
u_{i}^{p}\left(e_{i}^{h}, x_{-i}\right) & >u_{i}^{p}(x) \\
\sum_{h \in C\left(x_{i}\right)} x_{i h} u_{i}^{p}\left(e_{i}^{h}, x_{-i}\right) & >\sum_{h \in C\left(x_{i}\right)} x_{i h} u_{i}^{p}(x) \\
\Rightarrow u_{i}^{p}(x) & >u_{i}^{p}(x)
\end{aligned}
$$


And this contradiction completes the proof.

Lemma 2.3: $x$ is the fixed point of $D^{P}$ if and only if it is a Pareto Nash equilibrium.

Proof: We prove the first implication that if $x$ is a fixed point then $x$ is a Pareto Nash Equilibrium.

If $x$ is a fixed point then

$$
x_{i h}=\frac{x_{i h}+c_{i}^{h}(x)}{1+\sum_{k \in S_{i}} c_{i}^{k}(x)}, \quad \forall h \in S_{i}, \quad \forall i \in I
$$

By Lemma 2.2, $\exists h \in C\left(x_{i}\right)$ such that $u_{i}^{p}\left(e_{i}^{h}, x_{-i}\right) \leq u_{i}^{p}(x)$. For such a $h$, the above equation reduces to

$$
x_{i h}=\frac{x_{i h}}{1+\sum_{k \in S_{i}} c_{i}^{k}(x)}
$$

Since $x_{i h}>0$, we have $\sum_{k \in S_{i}} c_{i}^{k}(x)=0$. Since $c_{i}^{k}(x) \geq$ $0, \quad \forall k \in S_{i}$, we have $c_{i}^{k}(x)=0, \quad \forall k \in S_{i}, \quad \forall i \in I$. Then

$$
\begin{aligned}
& c_{i}^{k}(x)=0 \quad \forall k \in S_{i} \\
& \Rightarrow \text { Either one of the objective functions } \\
& u_{i}^{p}\left(e_{i}^{k}, x_{-i}\right)<u_{i}^{p}(x) \text { or } u_{i}^{p}\left(e_{i}^{k}, x_{-i}\right) \leq u_{i}^{p}(x), \forall p . \\
& \Rightarrow \exists \text { no } e_{i}^{k} \text { such that } \underline{u}_{i}\left(e_{i}^{k}, x_{-i}\right)>\underline{u}_{i}(x) \\
& \Rightarrow \exists \text { no } z \in \Delta_{i} \text { such that } \underline{u}_{i}\left(z, x_{-i}\right)>\underline{u}_{i}(x)
\end{aligned}
$$

Thus, $x$ is Pareto Nash equilibrium. Next, we prove the reverse implication that if $x$ is a Pareto Nash equilibrium, then $x$ is a fixed point of $D^{P}$.

Since $x$ is a Pareto Nash Equilibrium, $\forall i \in I, x_{i} \in \beta_{i}^{P}\left(x_{-i}\right)$,

$$
\begin{aligned}
& \Rightarrow \nexists z \in \Delta_{i} \text { such that } \underline{u}_{i}\left(z, x_{-i}\right)>\underline{u}_{i}(x) \\
& \Rightarrow \text { In particular there exists no } h \in S_{i} \\
& \text { such that } \underline{u}_{i}\left(e_{i}^{h}, x_{-i}\right)>\underline{u}_{i}(x) \\
& \Rightarrow c_{i}^{h}=0 \quad \forall h \in S_{i} \quad \forall i \in I .
\end{aligned}
$$

Therefore $x$ is a fixed point of the $D^{P}$.

Theorem 2.4: $\Theta^{P N E} \neq \emptyset$

Proof: The proof follows trivially from Lemma 2.1 and Lemma 2.3.

We trust that this proof method would inspire some dynamics to achieve the equilibria in the MOG setting. In the forthcoming sections, we develop a dynamics to reach a certain class of Pareto Nash equilibria.

\section{TWO PLAYER SYMMETRIC MULTI-OBJECTIVE GAMES}

Two player symmetric games have been widely studied in the game theoretic literature because they have been useful in modelling many social and economic interactions between homogeneous agents that perceive identical payoff functions [13]. In this regard, games such as Prisoner's dilemma, coordination game and stag hunt have been extensively studied. The notable property of these symmetric games is that they can be used to study an action strategy without associating it with any of the players. In this sense they are useful in characterizing the payoff of one action against another. This property makes two player symmetric games an attractive tool in large population games, where modelling the players becomes intractable.

In this paper, we extend these two player symmetric single objective games to MOGs. In particular, we are interested in characterizing the symmetric equilibria of these games. However, the same ideas can be extended to asymmetric equilibria. This is because there exists a polynomial-time reduction of the general Nash equilibria problem to the symmetric Nash equilibria problem [14]. However, it should be noted that the algorithmic reachability of the asymmetric equilibria in MOGs is non-trivial. These ideas will be discussed in the forthcoming sections.

Since we consider two player games $(|I|=n=2)$ in greater detail, we introduce the notion of Multiple Matrix Games (MMG), which is a natural extension of the $\mathrm{Bi}$ Matrix Games in the single objective setting. The same matrix notation has also been considered by [12]. Associated with each player is a sequence of matrix payoff functions $\left(A_{1}, A_{2}, \cdots, A_{l_{1}}\right)$ and $\left(B_{1}, B_{2}, \cdots, B_{l_{2}}\right)$ (for player $I$ and $I I$ respectively). We assume that player $I$ is the row-player and player II is the column player. Let us suppose that player $I$ chooses strategy $\xi \in \Delta_{1}$ and player II chooses strategy $\eta \in \Delta_{2}$. Then, the expected scalar payoff with respect to the $p^{t h}$ and $q^{t h}$ objectives of the MOG for player $I$ and $I I$ is given by $\xi^{T} A_{p} \eta$ and $\xi^{T} B_{q} \eta$ respectively, where $p \in\left\{1,2, \cdots, l_{1}\right\}$ and $q \in\left\{1,2, \cdots, l_{2}\right\}$.

The analysis in this paper concerns symmetric two player games. Since the games are symmetric we can denote both players' pure strategy set as $K=\{1,2, \cdots, k\}$, and the associated mixed strategy set as $\Delta=\left\{x \in \mathbb{R}_{+}^{k}: \sum_{i \in K} x_{i}=\right.$ $1\}$. In these games $\left(B_{1}=A_{1}^{T}, B_{2}=A_{2}^{T}, \cdots, B_{l}=A_{l}^{T}\right)$. The polyhedron of mixed strategy profiles is $\Theta=\Delta^{2}$. The vector payoff to strategy $x \in \Delta$ when played against $y \in \Delta$ is given by $\underline{u}(x, y)=\left(u^{1}, u^{2}, \cdots, u^{l}\right)(x, y)=$ $\left(x . A_{1} y, x . A_{2} y, \cdots, x . A_{l} y\right)$. The Pareto best response replies to any strategy $y \in \Delta$ is denoted by $\beta^{P *}(y) \subset \Delta$. The set of all symmetric Pareto Nash equilibria is denoted by $\Delta^{P N E}$.

\section{A. SYMMETRIC EQUILIBRIA IN SYMMETRIC GAMES}

The set of Pareto Nash equilibria for the vector payoff symmetric two player game are given by

$$
\begin{aligned}
\Delta^{P N E} & =\left\{x \in \Delta \quad \mid \quad x \in \beta^{P *}(x)\right\} \\
& =\left\{x \in \Delta \quad \mid x=\arg \max _{w \in \Delta} \underline{u}(w, x)\right\}
\end{aligned}
$$

where max stands for Pareto maximization.

There is an alternate characterization of symmetric Pareto Nash equilibria which is developed from multi-criteria linear programming (MCLP). [11] and [17] introduce the terminology and definitions related to MCLP in a MOG. In order to establish the relation with MCLP, we begin with characterizing the geometry of the $\beta^{P *}$. Let us denote the payoff space against strategy $x \in \Delta$ as $\mathcal{U}(x)$. Let $\Delta_{E}$ denote the set of all efficient strategies. The corresponding set of non-dominated points in $\mathcal{U}$ is denoted by $\mathcal{U}_{N}$.

Since both the decision space $\Delta$ and the objective space $\mathcal{U}(x)$ are finite dimensional we can associate an objective 
matrix $U$. $U$ will be parameterized by the opposing strategy $x \in \Delta$. Thus, the symmetric Pareto Nash problem can be reformulated and posed as

$$
\max _{w \in \Delta}^{P} U w
$$

Let $\mathbb{R}_{>0}^{l}$ denote the set of $l$-dimensional vectors such that for $a \in \mathbb{R}_{>0}^{l}, a>\underline{0}$, where $>$ is the component-wise order for vectors.

Definition Let $z \in \Delta_{E}$. If there is some $\underline{\lambda} \in \mathbb{R}_{>0}^{l}$ such that $z$ is an optimal solution of $\max \underline{\lambda}^{T} U w$, then $z$ is called a supported efficient strategy and $U z$ is called supported nondominated payoff against $x \in \Delta$. The set of all supported efficient strategies and supported non-dominated payoffs are denoted by $\Delta_{s E}$ and $\Delta_{s N}$.

The weights $\underline{\lambda} \in \mathbb{R}_{>0}^{l}$ signify the trade-off between the various objectives of the vector payoff. We next present a sequence of lemmas that characterize the efficient strategies and non-dominated points. Supporting arguments to these lemmas are found in [11].

Lemma 3.1: $\Delta_{E} \neq \emptyset$

Proof: $\Delta$ is a closed set. The payoff set $\mathcal{U}=\{U w \mid w \in$ $\Delta\}$ is also thus a closed set (by continuity of the bounded linear operator). Hence $\mathcal{X}_{i E} \neq \emptyset$.

Lemma 3.2: $\Delta_{E}=\Delta_{s E}$ and $\mathcal{U}_{N}=\mathcal{U}_{s N}$.

Proof: $\Delta$ is a convex set.

$\Rightarrow\{U w \mid w \in \Delta\}$ is a convex set. $\Rightarrow \mathcal{U}_{s N}=\mathcal{U}_{s N}$. (Theorem 3.5 of [11]).

Lemma 3.2 suggests that the Pareto reply can be solved by weighted sum scalarization. For reference on this technique, we recommend Chapter 3 of [11]. For the symmetric Pareto Nash equilibrium, we show that the weights can be constructed very efficiently using MCLP simplex methods. For a detailed reference on these topics, we refer the reader to Chapter 2 of [15] and Chapters 7 and 8 of [11].

Definition The weighted sum linear program, for $\underline{\lambda} \in \mathbb{R}^{l}$, corresponding to the $\beta^{P *}$ in equation (1) is

$$
\max _{w \in \Delta} \underline{\lambda}^{T} U w
$$

Let us denote the set of solutions for the problem posed in Equation (2) by $L P_{\text {soln }}(\lambda)$.

Lemma 3.3: $\cup_{\underline{\lambda} \in \mathbb{R}_{>0}^{l}} L P_{\text {soln }}(\underline{\lambda})=\beta^{P *}$

Proof: The proof follows trivially from Lemma 3.2.

Lemma 3.4: The payoff space $\mathcal{U}$ is supported by a finite set of hyperplanes.

Proof: $\Delta$ is a convex compact set. If $\underline{u}$ is a bounded function for every objective, then $\mathcal{U}_{i}\left(x_{-i}\right)$ is a convex polytope. A convex polytope has a finite number of faces and each face is supported by a hyperplane (Chapter 2 of [15]).

Theorem 3.5: There exists a finite set $\Lambda=\left\{\underline{\lambda} \in \mathbb{R}_{>0}^{l}\right\}$ such that $\cup_{\underline{\lambda} \in \Lambda} L P_{\text {soln }}(\underline{\lambda})=\beta^{P *}(x)$

Proof: The existence proof is a direct implication from Lemmas 3.3 and 3.4 .
It is clear that the complexity of computing the symmetric equilibria is reduced to that of computing the tradeoff weights $\underline{\lambda}$. In the next section, we present very efficient algorithms to compute these weights.

\section{SELECTING TRADEOFF WEIGHTS}

In this section, we establish constructively that there are only a finite set $\Lambda$ of non-degenerate weights, which yield all the non-dominated points. We use simplex methods for parametric linear programs [11]. First, we present the simplex algorithm to obtain the trade-off weights for bi-objective games. In the section to follow, we show its extension to a general MOG.

\section{A. BI-OBJECTIVE GAMES}

For a bi-objective game, the Pareto problem in Equation (1) can be stated as

$$
\begin{aligned}
\operatorname{P} & \left(U^{1} w, U^{2} w\right) \\
\text { subject to } & \underline{1}^{T} w=1 \\
& w \geq 0
\end{aligned}
$$

where $U^{1}$ and $U^{2}$ are the first and second rows of $U$ respectively. Let us consider a parametric objective $(\lambda \in$ $[0,1])$

$$
u(\lambda)=\lambda U^{1}+(1-\lambda) U^{2}
$$

This yields a parametric linear program

$$
\max \left\{u(\lambda) w: \underline{1}^{T} w=1, w \geq 0\right\} .
$$

Now we can construct the simplex algorithm. For any feasible basis $\mathcal{B}$, let the vector of reduced gains be denoted by

$$
\bar{u}(\lambda)=\lambda \bar{U}^{1}+(1-\lambda) \bar{U}^{2} .
$$

where $\bar{U}^{1}$ and $\bar{U}^{2}$ are reduced cost vectors (defined in Chapter 6 of [11]). Let us denote the non-basic indices by $\mathcal{N}$. If $\bar{u}(\lambda) \leq 0$, then the simplex algorithm achieves optimality of the parametric linear program and terminates (Chapter 6 of [11]). If $\bar{U}^{2} \leq 0$, then $\bar{u}(\lambda) \leq 0$ for all $\lambda>\hat{\lambda}$. This simplex method is illustrated in Algorithm 1. The algorithm returns the list $\mathcal{L}$ of non-degenerate weights.

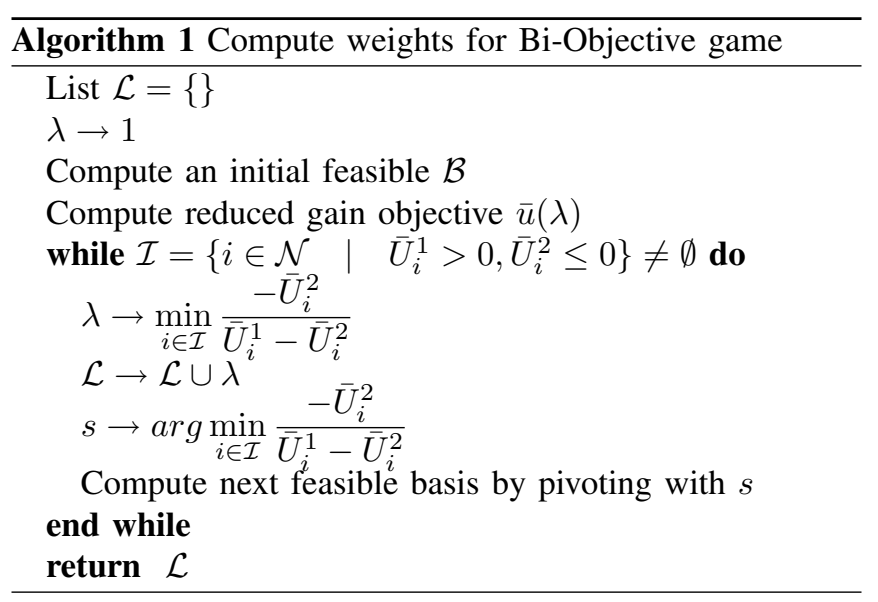




\section{B. MULTI-OBJECTIVE GAMES}

We extend Algorithm 1 to the MOG case. Algorithm 2 is an abstraction of Algorithms 6.2 and 7.1 of [11]. By transversing through the connected efficient bases, we obtain the kissing planes of the $\mathcal{U}$. The number of efficient pivots is finite and hence $\Lambda=\mathcal{L}$.

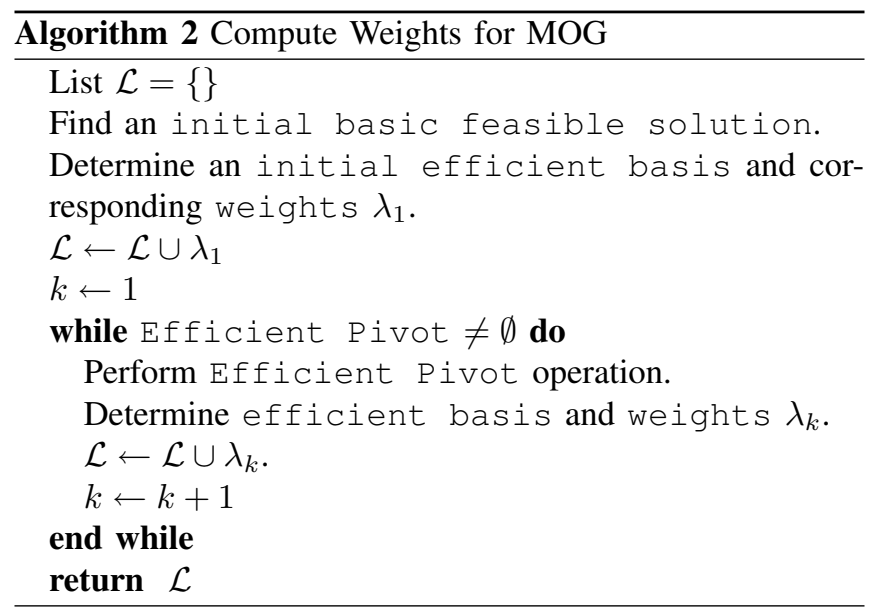

Once these algorithms return the set of weights, the next step is to achieve the various symmetric equilibria. In this paper, we adopt an evolutionary dynamics to achieve the equilibria.

\section{BIASED REPLICATOR DYNAMICS}

The ideas of Maynard Smith and Price [16] on evolutionary robustness gave rise to an evolutionary dynamics called the replicator dynamics. These dynamics have several interesting dynamic stability properties that are related to the Nash equilibrium of a corresponding auxiliary game. Chapters 3 and 5 of [10] include a survey of these properties.

We provide an extension of the replicator dynamics to multi-objective games, and the proofs of dynamic stability of this extension. We also establish the relationship between the dynamic stable equilibria of this extension and the Pareto Nash equilibria.

\section{A. EVOLUTIONARY STABILITY}

In this subsection, we introduce the notion of evolutionary stability for multi-objective large population games. Assume, there exists a large population that plays the same incumbent strategy $x \in \Delta$. Suppose, a small group of mutants, which are programmed to play some mutant strategy $y \in \Delta$, are introduced into the original population. Let the share of mutants in the post-entry population be $\epsilon \in(0,1)$. Pairs of individuals are drawn from this hybrid population to play a MOG. For any individual, the probability that the opponent will play the mutant strategy $y$ is $\epsilon$, and the probability that the opponent will play the incumbent strategy $x$ is $1-\epsilon$. Thus, the post-entry payoff to the incumbent strategy is $\underline{u}(x, \epsilon y+(1-\epsilon) x)$, and that to the mutant strategy is $\underline{u}(y, \epsilon y+(1-\epsilon) x)$.
Definition A strategy $x \in \Delta$ is said to be evolutionary stable in the $\succ$ ordering if for $y \in \Delta$ and $y \neq x$, there exists $\epsilon_{y}$ such that $\forall \epsilon \in\left(0, \epsilon_{y}\right)$

$$
\underline{u}(x, \epsilon y+(1-\epsilon) x) \succ \underline{u}(y, \epsilon y+(1-\epsilon) x) .
$$

In the above definition, $\succ$ can be one of the several vector orders. In this work we consider the $\lambda$-scalarized order, i.e., $\underline{a}>\lambda \underline{b}$, for $x, y \in \mathbb{R}^{l}$, if and only if $\underline{\lambda}^{T} \underline{a}>\underline{\lambda}^{T} \underline{b}$, for $\underline{\lambda}>0$.

\section{B. BIASED EVOLUTIONARY STABILITY}

The $\underline{\lambda}$-scalarized order gives rise to, what we call, the Biased Evolutionary Stability. To characterize the biased evolutionary stable strategies, consider an auxiliary symmetric single objective game $G(\underline{\lambda})$. The payoff function for strategy $x \in \Delta$ against $y \in \Delta$ in game $G(\lambda)$ is given by $\underline{\lambda}^{T} \underline{u}(x, y)$ (where $\underline{u}(x, y)$ is the corresponding vector payoff in the multi-objective game).

Proposition 5.1:

$\Delta^{B E S S}=\left\{x \in \Delta^{P N E} \quad \mid y \neq x, y \in \beta^{* P}(x) \Rightarrow\right.$ $x$ dominates $y$ as a reply to opponent strategy $y\}$

Proof:

The proof is based on the score function of the auxiliary game.

$$
\begin{array}{ll} 
& f(\epsilon, y)=\lambda^{T} \underline{u}(x-y, x)+\epsilon \lambda^{T} \underline{u}(x-y, y-x)>0 \\
\Rightarrow \quad & \lambda^{T} \underline{u}(x-y, x) \geq 0 \\
\Rightarrow \quad & \lambda^{T} \underline{u}(x, x) \geq \lambda^{T} \underline{u}(y, x) \\
\Rightarrow \quad & x \in \Delta^{P N E} \quad(\text { by } 3.2) \\
& \text { if } y \in \beta^{* P}(x) \Rightarrow \lambda^{T} \underline{u}(x-y, y-x)>0 \\
& \Rightarrow \lambda^{T} \underline{u}(x, y)-\lambda^{T} \underline{u}(y, y)<0 \\
& \Rightarrow x \text { dominates y as a reply to strategy } y
\end{array}
$$

\section{POPULATION DYNAMICS UNDER BIASED FITNESS FUNCTION}

Consider a large but finite population of individuals who are programmed to play the pure strategies $i \in K$ in a symmetric multi-objective two player game with the vector payoff function $\underline{u}$. Let $p_{i}(t) \geq 0, \quad t \geq 0$, denote the number of individuals who are currently programmed to the pure strategy $i \in K$, and $p(t)=\sum_{i \in K} p_{i}(t) \geq 0$. The population state is given by the population share vector $x(t)=\left(x_{1}(t), x_{2}(t), \cdots, x_{k}(t)\right)$, where each component $x_{i}(t)=p_{i}(t) / p(t)$. Thus $x(t) \in \Delta$. The expected vector payoff to any pure strategy $i$ at a random match, when the population is in state $x \in \Delta$, is $u\left(e^{i}, x\right)$. The associated population average payoff is given by

$$
\underline{u}(x, x)=\sum_{i=1}^{k} x_{i} \underline{u}\left(e^{i}, x\right) .
$$

It is common in evolutionary biology to describe the population dynamics using a fitness function. The fitness 
function usually describes the number of offsprings per unit time. A particular population changes its population share based on its fitness in the current population state. For this work, we assume that fitness is governed by a biased payoff function. Then, the corresponding dynamics for the population shares $x_{i}$ becomes

$$
\dot{x_{i}}=\left[\underline{\lambda}^{T} \underline{u}\left(e^{i}, x\right)-\underline{\lambda}^{T} \underline{u}(x, x)\right] x_{i} \quad \cdots(B R E(\underline{\lambda}))
$$

\section{DYNAMIC STABILITY OF BRE}

There is an alternative representation for the Pareto Nash Equilibria given by the following lemma. $\mathcal{U}$ is a convex polyhedron with vertices in $u\left(e^{i}, x\right), \quad i \in K$.

\section{Lemma 5.2:}

$$
\begin{aligned}
& \Delta^{P N E} \\
& =\left\{x \in \Delta \mid \underline{\lambda}^{T} \underline{u}\left(e^{i}, x\right)=\underline{\lambda}^{T} \underline{u}(x, x), i \in C(x),\right. \text { and } \\
& \underline{u}\left(e^{i}, x\right) \in \text { Pareto Dominating Face of } \mathcal{U}, \\
& \text { where } \underline{\lambda} \in \mathcal{R}_{>0}^{l} \\
& \text { is the normal to the corresponding face. }\}
\end{aligned}
$$

Proof: Every face of the convex polyhedron is supported by a hyperplane, and thus there exists $\underline{\lambda} \in \mathbb{R}_{>0}^{l}$ such that $\underline{\lambda}^{T}\left(\underline{u}\left(e^{i}, x\right)-\underline{u}(y, x)\right)=0$ for all $y$ in a particular face of the convex polyhedron. If the face of the polyhedron contains $x$, then $\underline{\lambda}^{T}\left(\underline{u}\left(e^{i}, x\right)-\underline{u}(x, x)\right)=0$. Further, if $x \in$ Pareto dominating face of the convex polyhedron then $x \in \Delta^{P N E}$.

\section{E. STATIONARY STATES}

The stationary states of the autonomous dynamics of $B R E(\underline{\lambda})$ are given by

$$
\Delta^{o}(\underline{\lambda})=\left\{x \in \Delta \mid \underline{\lambda}^{T} \underline{u}\left(e^{i}, x\right)=\underline{\lambda}^{T} \underline{u}(x, x) \quad \forall i \in C(x)\right\}
$$

Let $\Delta^{o o}(\underline{\lambda})$ denote the set of interior stationary states of $\operatorname{BRE}(\underline{\lambda})$, i.e., $\Delta^{o o}(\underline{\lambda})=\Delta^{o}(\underline{\lambda}) \cap \operatorname{int}(\Delta)$.

Proposition 5.3: $\left\{e^{1}, e^{2}, \cdots, e^{k}\right\} \cup \Delta^{P N E}(\underline{\lambda}) \subset \Delta^{o}(\underline{\lambda})$. $\Delta^{o o}(\lambda)=\Delta^{P N E}(\lambda) \cap \operatorname{int}(\Delta)$.

Proposition 5.4: If $x \in \Delta$ is Lyapunov stable in $B R E(\underline{\lambda})$, then $x \in \Delta^{P N E}(\underline{\lambda})$.

Let us suppose that $\zeta_{\underline{\lambda}}\left(t, x_{0}\right)$ is a solution to $B R E$ with $\zeta_{\underline{\lambda}}\left(0, x_{0}\right)=x_{0}$.

Proposition 5.5: If $x_{0} \in \operatorname{int}(\Delta)$ and $\zeta\left(t, x_{0}\right) \rightarrow x$, then $x \in \Delta^{P N E}(\underline{\lambda})$.

The proofs for Propositions 5.3-5.5 for the auxiliary single objective game, $G(\underline{\lambda})$, are given in Chapter 3 of [10]. We proceed to characterise the asymptotically stable states of the dynamics. We begin by introducing a candidate Lyapunov function . To define its domain, consider the neighbourhood set

$$
Q_{x}=\{y \in \Delta \mid \quad C(x) \subset C(y)\} .
$$

The function $H_{x}: Q_{x} \rightarrow \mathbb{R}$

$$
H_{x}(y)=\sum_{i \in C(x)} x_{i} \log \left(\frac{x_{i}}{y_{i}}\right)
$$

behaves as a Lyapunov function for the biased replicator dynamics [10]. The time derivative along the dynamics is given by

$$
\left.H_{x} \dot{(} y\right)=-\underline{\lambda}^{T}[\underline{u}(x, y)-\underline{u}(y, y)]
$$

Theorem 5.6: If $x \in \Delta^{B E S S}$ then $B R E(\underline{\lambda})$ is asymptotically stable.

Proof: The proof is based on Lyapunov's direct method. Since $x \in \Delta^{B E S S}$, we have $H_{x}(y)<0 \quad \forall y \in N_{x} \cup Q_{x}$, where $N_{x}$ is some neighbourhood of $x$.

\section{CONCLUSIONS}

We have presented an alternate proof method that establishes the existence of equilibria for MOGs. We have shown an extension of evolutionary stability to these MOGs. As with single objective games, we have shown that a modified form of replicator dynamics achieves these evolutionarily stable strategies. We have also shown that at these equilibria, the tradeoff weights among various payoffs can be efficiently computed using simplex methods of MCLP.

\section{ACKNOWLEDGEMENT}

This material is based upon work supported by the Communications and Networks Consortium sponsored by the U.S. Army Research Laboratory under the Collaborative Technology Alliance Program, Cooperative Agreement DAAD19-01-2-0011 and the MURI Award Agreement W911-NF-0710287 from the Army Research Office.

\section{REFERENCES}

[1] El-Azouzi R. Jimenez T. Altman E., Boulogne T. and Wynter L. A survey on networking games in telecommunications. Computers and Operations Research, 2006.

[2] D. Blackwell. An analog of the minimax theorem for vector payoffs. Pacific Journal of Mathematics, 6(1):1-8, 1956.

[3] Contini B.M. A decision model under uncertainity with multiple payoffs. In In A. Mensch, ed., Theory of Games; Techniques and Applications, pages 50-63. American Elsevier Pub. Co., 1966.

[4] Rousseau J.J. Charnes A., Huang Z.M. and Wei Q.L. Corn extremal solutions of multi-payoff games with cross-constrained strategy set. Optimization, 21(1):51-69, 1990.

[5] Zelikovsky A. Dmitrii L., Solomon D. Multiobjective games and determining pareto-nash equilibria. BULETINUL ACADEMIEI DE STIINTE, A REPUBLICII MOLDOVA MATEMATICA, (3):115-122, 2005.

[6] Granas A. Dugundji J. Fixed Point Theory. Springer, 2003.

[7] Nash J. Non-cooperative games. PhD thesis, Princeton University, May 1950.

[8] Nash J. Non-cooperative games. Ann. Math., 54:286-295, 1951.

[9] Zhao J. The equilibria of a multiple objective game. International Journal of Game Theory, 20:171-182, 1991.

[10] Weibull J.W. Evolutionary Game Theory. MIT Press, August 1997.

[11] Ehrgott M. Multicriteria Optimization. Springer, 2 edition, 2005.

[12] Zeleny M. Games with multiple payoffs. International Journal of Game Theory, 4(4):179-191, December 1975.

[13] Osborne M.J. An Introduction to Game Theory. Oxford University Press, USA, 2003.

[14] Tardos E. Nisan N., Roughgarden T. and Vazirani V.V., editors. Algorithmic Game Theory. Cambridge University Press, 2007.

[15] Steiglitz K. Papadimitriou C. H. Combinatorial Optimization Algorithms and Complexity. Dover, 1998.

[16] Price G. R. Smith M. The logic of animal conflict. Nature, 246:15-18, 1973.

[17] Baras J.S. Somasundaram K. Pareto nash replies for multiobjective games. Technical report, Institute for Systems Research, http://hdl.handle.net/1903/8937, 2008. 